\title{
Sinnverneinung. Warum der assistierte Suizid uns alle angeht
}

\author{
Roland Kipke
}

Eingegangen: 12. April 2021 / Angenommen: 14. Juli 2021 / Online publiziert: 7. September 2021

(C) Der/die Autor(en) 2021

Zusammenfassung Der Artikel beantwortet die Frage nach der ethischen Bewertung des assistierten Suizids auf neue Weise: indem er die Verneinung von Lebenssinn, wie sie sich in Suiziden oft ausspricht, in den Mittelpunkt rückt. Anders als üblich fasst er die Sinnfrage nicht als bloß individualethische, sondern als sozialethische Angelegenheit auf. Denn der nahezu unvermeidbaren Orientierung am Ziel eines sinnvollen Lebens ist eine grundlegende soziale Verbindung eingeschrieben: eine implizite Wertschätzung von Menschen als potenziellen Sinnquellen und als Subjekten von Urteilen über Lebenssinn. Suizide neigen dazu, diese Verbindung zu negieren. Dieses Verständnis des Suizids kann eine Reihe von evaluativen und normativen Annahmen aufklären und begründen, die in der Debatte um den assistierten Suizid eine prominente Rolle spielen.

Schlüsselwörter Assistierter Suizid · Suizidprävention · Sinn · Sinnvolles Leben · Menschenwürde $\cdot$ Autonomie

\section{The denial of meaning in life. Why assisted suicide concerns us all}

\begin{abstract}
Definition of the problem The ethical debate about assisted suicide remains controversial and is also based in part on assumptions that are taken for granted, but which, on closer inspection, lack justification.
\end{abstract}

Dr. Roland Kipke $(\bowtie)$

Abteilung Philosophie, Universität Bielefeld, 33501 Bielefeld, Deutschland

E-Mail: r.kipke@uni-bielefeld.de 
Arguments The article develops a new approach by focusing on the social dimension of the denial of meaning in life, which is often expressed by suicides. For a fundamental social connection is included in the human orientation towards the goal of a meaningful life, namely an implicit appreciation of human beings as potential sources of meaning and as subjects of judgments about the meaningfulness. Suicides tend to negate this connection.

Conclusion This view of suicide can, among other things, help to adquately understand the meaning of (assisted) suicide and justify the widely shared assumptions of a primacy of suicide prevention and a conscientious objection right of doctors.

Keywords Assisted suicide $\cdot$ Suicide prevention $\cdot$ Meaningfulness $\cdot$ Meaningful life $\cdot$ Human dignity $\cdot$ Autonomy

\section{Einleitung}

Was hat der ärztlich assistierte Suizid mit dem Sinn des Lebens zu tun? Eine Antwort liegt nahe: Menschen, die Suizid begehen wollen, sehen keinen Sinn mehr in ihrem Leben. Doch diese Feststellung ist anscheinend nicht sonderlich interessant, weil wohlbekannt. Zudem scheint sie für die Frage nach der ethischen Bewertung der Suizidassistenz nicht direkt von Belang zu sein. Denn die Zuschreibung von Lebenssinn gilt weithin als etwas, das allein der jeweiligen Person zusteht, als Angelegenheit der autonomen Entscheidung also, und diese individuelle Autonomie ist bei der Frage der Zulässigkeit von Suizidassistenz moralisch und rechtlich ausschlaggebend (vgl. Gutmann 2002, S. 172f., 181; BVerfG 2020, Rn. 209 f.; Borasio et al. 2020; Wittwer 2020). ${ }^{1}$ Die Sinnthematik wäre demnach zwar persönlich wichtig, aber sozialethisch irrelevant.

Ich vertrete hier die gegenteilige These: Sinn ist nicht etwas, das allein die individuelle Person etwas angeht, sondern etwas, das uns verbindet. Das ist nicht die triviale Behauptung, dass wir in unseren Überzeugungen von dem, was ein Leben sinnvoll macht, von gesellschaftlichen Vorstellungen geprägt sind. Gemeint ist eine stärkere These: In unserer Orientierung am Ideal eines sinnvollen Lebens sind wir auf aufeinander als Sinnquellen bezogen und auf die Sinnbejahung durch andere Menschen angewiesen. Deshalb betrifft die Sinnverneinung eines suizidenten Menschen nicht diesen allein, sondern uns alle.

Das mag zunächst abwegig, ja gefährlich klingen. Abwegig, weil wir wie gesagt gewohnt sind, Sinnsuche und Sinnfindung als etwas nur Individuelles zu verste-

\footnotetext{
1 Autonomie verstehe ich hier im Sinne des Standardmodells der Medizinethik in erster Linie als absichtliche, freiwillige und informierte Entscheidungen über persönliche Angelegenheiten (vgl. Beauchamp und Childress 2019, Kap 4). Obschon im Kern individualistisch, muss dieses Autonomieverständnis keineswegs die soziale Einbettung des Individuums ignorieren (vgl. Ach und Schöne-Seifert 2013). Allerdings bezieht sich das Standardmodell nur ,lokal“ auf einzelne Handlungen, während bei autonomen Entscheidungen für einen Suizid in stärkerem Maße übergreifende Lebensbewertungen hineinspielen, als dies bei vielen Entscheidungen im medizinischen Alltag der Fall ist. Insofern ist das hier zugrunde gelegte Autonomieverständnis offen für eine Erweiterung in Richtung personaler Autonomie. „Autonomie“/,autonom“ verwende ich synonym mit „Selbstbestimmung“/,,selbstbestimmt“.
} 
hen. Gefährlich, weil Entscheidungen über die Sinnhaftigkeit eines Lebens allein den einzelnen Patienten zuzugestehen eine zentrale Errungenschaft der medizinethischen Moderne ist. Daher wird man leicht die Rückkehr eines harten Paternalismus wittern, wenn Sinnfragen nicht nur als individuelle Angelegenheit angesehen werden. Beim Thema Suizid kommt hinzu, dass vielen Menschen vor Augen steht, wie suizidente Menschen früher auf empörende Weise zusätzlich zu ihrer Verzweiflung mit moralischer Verachtung bestraft wurden.

Doch hinter solchen Bedenken steht eine allzu simple Koppelung von Autonomieachtung und Argwohn gegenüber überindividuellen Maßstäben der Lebensbewertung. Auf eine solche konzeptionelle Kopplung aber ist ein liberaler Standpunkt nicht angewiesen. Personen in ihren persönlichen Angelegenheiten für allein entscheidungsbefugt zu halten, impliziert nicht, auf jeden übersubjektiven Maßstab der Beurteilung ihrer Entscheidungen verzichten zu müssen. Achtung der Patientenautonomie bedeutet nicht, dass jede autonome Entscheidung klug und die zugrundeliegende Bewertung richtig ist. Und individuelle Suizidentscheidungen auch für eine soziale Angelegenheit zu halten, heißt nicht, sie der Entscheidungsvollmacht des Individuums entziehen zu wollen. Dementsprechend trägt auch die These dieses Aufsatzes keine paternalistische Tendenz in sich.

Wenn auch dieser erste Einwand nicht gegen den hier zu entwickelnden Ansatz spricht, was spricht für ihn? Hier seien vorab einige Vorzüge thesenhaft genannt: Der Ansatz kann den widersprüchlichen Gebrauch der Menschenwürdeidee im Diskurs um den assistierten Suizid verständlich machen. Er kann überhaupt erst für eine angemessene Interpretation des Phänomens Suizid sorgen - was in der Rechtebasierten ethischen Diskussion oft unter den Tisch fällt. Damit kann er auch die sehr unterschiedlichen Urteile über verschiedene Suizidmotive verständlich machen. Der Ansatz vermag des Weiteren zu begründen, warum der Suizidprävention auch bei autonomen Suizidwünschen ein moralisches Primat zukommt, wie es allenthalben angenommen, aber selten ethisch begründet wird. Schließlich kann er eine tragfähige Begründung dafür liefern, warum Ärzte das Recht haben sollen, eine Suizidassistenz $\mathrm{zu}$ verweigern.

So gehe ich vor: Zunächst erkläre ich, was unter Sinn zu verstehen ist und wie wichtig er ist. Darauf aufbauend entwickle ich das Verständnis der sinnkonstituierten Verbundenheit und skizziere eine darauf bauende Konzeption von Menschenwürde. Die Suizidproblematik ist in diesem Abschnitt bereits im Blick, aber in den Mittelpunkt rückt sie erst im nächsten Abschnitt. Hier übertrage ich die Überlegungen zur sinnkonstituierten Verbundenheit auf den Suizid und begründe so die zentrale These, dass der assistierte Suizid aufgrund der damit verknüpften Sinnverneinung ein Problem von gesellschaftlicher Relevanz ist. Schließlich diskutiere ich die Vorzüge dieses Ansatzes.

Ein Wort noch zur Begrifflichkeit: Auch wenn im medizinethischen Kontext vor allem der ärztlich assistierte Suizid Thema ist, spreche ich allgemein vom assistierten Suizid, weil die Überlegungen ebenso für nicht-ärztliche Suizidassistenz gelten. 


\section{Sinn}

Beim Thema Sinn gibt es ein Problem. Kulturell einflussreiche Vorstellungen von Sinn erschweren die Bereitschaft für eine rationale Auseinandersetzung damit. Gemeint sind vor allem Vorstellungen von einem letzten Zweck, der dem menschlichen Dasein vorgegeben sei. Solche kosmologisch-teleologischen Konzeptionen sind oft mit der traditionellen Rede vom „Sinn des Lebens“ verbunden. Das Problem dieser Konzeptionen ist offensichtlich: Ein letzter Zweck ließ sich bislang nicht entdecken, und denkbar ist er nur unter der Annahme einer zwecksetzenden Instanz oder einer teleologisch verfassten Natur. Weil solche Annahmen unweigerlich in metaphysisch vermintes Gelände führen, stößt die Rede vom Sinn des Lebens im akademischen Kontext zuweilen - und verständlicherweise - auf skeptisches Stirnrunzeln und nihilistisches Achselzucken.

Glücklicherweise sind solche Konzeptionen nicht die einzige Möglichkeit, Sinn in Bezug auf menschliches Leben zu verstehen. Sie stehen auch im gegenwärtigen philosophischen Sinndiskurs nicht in hohem Ansehen. Mit der großen Mehrheit in diesem Diskurs verstehe ich unter „Sinn“ nicht etwas Monolithisches und Vorgegebenes, sondern etwas, das sich je nach Person unterscheidet und von ihrem Handeln und ihrer Lebensweise abhängt (vgl. Wolf 2010; Metz 2015; Landau 2017). Dementsprechend ist Sinn gradualisierbar, ein Leben kann mehr oder weniger sinnvoll sein. Statt von „dem Sinn des Lebens“ sprechen viele Autoren heute lieber vom „Sinn im Leben“ oder einfach vom „sinnvollen Leben“ (bzw. „meaning in life“ und „meaningful life“, (vgl. Wolf 2010; Metz 2015; Landau 2017)).

Wodurch aber gewinnt ein Leben an Sinn? Viele philosophische Ansätze ebenso wie lebensweltliche Intuitionen lassen sich durch folgendes Verständnis abdecken (vgl. Rüther 2021): Das Leben einer Person ist sinnvoll, insofern sie in positiver Weise und aktiv auf intrinsisch Wertvolles außerhalb ihrer selbst bezogen ist. „In positiver Weise" bedeutet, dass diese sinnstiftende Beziehung von bejahender Art ist, also schaffend, liebend, pflegend, schützend, rettend, verbessernd usw. „Aktiv““ heißt, dass dabei ein Mindestmaß an Aktivität notwendig ist. Reine Widerfahrnisse sind ausgeschlossen, die Person muss als Person beteiligt sein. Wie sich, ,intrinsische Werte“ philosophisch begründen lässt, ist umstritten, doch wir alle sind mit solchen Wertungen vertraut: Wir schätzen etwas nicht wegen seines Nutzens, sondern um seiner selbst willen. Das können Menschen sein, aber ebenso Erkenntnisse, Kunstwerke und vieles mehr. „Außerhalb ihrer selbst“" meint schlicht, dass es nicht die eigene Person ist, auf die positiv bezogen zu sein Sinn stiften kann. Selbstverliebtheit schafft keinen Sinn.

Was zunächst abstrakt und formelhaft wirken mag, entspricht dem, was weithin als sinnvoll gilt. Für die meisten Menschen dürften sinnstiftende Verbindungen vor allem zu anderen Menschen bestehen, seien es Freunde, Lebenspartner oder Kinder, aber auch Patienten, Kollegen, Vereinsmitglieder und viele mehr. Ebenso kann ein Leben durch wissenschaftliche, künstlerische oder humanitäre Aktivitäten an Sinn gewinnen. Ob die Pflege guter Freundschaften, eine altruistische Nachbarschaftshilfe, ein gemeinwohlorientiertes politisches Engagement, die Pflege demenzkranker Menschen, das Schaffen inspirierender bildhauerischer Werke, der Schutz bedrohter Vogelarten, eine psychotherapeutische oder pädagogische Arbeit, die Gewinnung 
astrophysikalischer oder medizinethischer Einsichten - das Spektrum sinnvollen Lebens ist weit und bunt.

Sinn wird zwar durch ein Individuum gestiftet, ist aber nicht bloß subjektiver Natur. Das heißt, was intrinsisch wertvoll ist, bestimmt sich nicht nach dem Dafürhalten der jeweiligen Person. Ein reiner Sinn-Subjektivismus hätte extrem kontraintuitive Konsequenzen und wird deshalb auch von der großen Mehrheit der heutigen Autoren und Autorinnen abgelehnt (vgl. Wolf 2010; Metz 2015; Landau 2017). Denn dann wäre eine Tätigkeit sinnvoll, sobald die betreffende Person sie gerne ausübt oder für sinnvoll hält. Die Demokratisierung eines autoritären Regimes wäre genauso sinnvoll wie Grashalme im Vorgarten zu zählen; die Schaffung eines bahnbrechenden literarischen Werks genauso wie ein leidenschaftliches, aber dilettantisches Geschreibsel.

Selbstverständlich ist der Streit darüber notorisch, was im Einzelnen objektiv intrinsisch wertvoll ist und nicht nur subjektiv so empfunden wird. Doch dass wir darüber diskursiv streiten, zeigt, dass es auf diesem Feld rationale Gründe gibt, die für das eine und gegen etwas anderes sprechen. Hier ergeht es uns nicht anders als bei moralischen Fragen: Wir mögen uns uneinig sein, was im Einzelnen richtig und falsch ist, aber wir sind uns in der Regel darüber einig, dass es ein solches Richtig und Falsch gibt.

Soweit zu einer knappen Bestimmung dessen, was Sinn ist und was ein sinnvolles Leben ausmacht. Zu klären ist noch die praktische Bedeutung, die Sinn für uns hat. Sie ist kaum zu überschätzen. Ob man den Begriff ,Sinn“ verwendet oder nicht, das damit Gemeinte ist es, was unserem Leben evaluatives Gewicht verleiht. Auf dieses Gewicht sind wir existentiell angewiesen. Durch seinen Beziehungscharakter hebt Sinn uns gewissermaßen aus der Kleinheit des eigenen Ich und verschafft unserem Leben Wert, Relevanz, Bedeutsamkeit. Auch das Glück, das im Sinne von Erfülltheit und langfristiger Zufriedenheit oft als höchster Wert des Lebens verstanden wird, hängt maßgeblich von Sinnerfahrung ab. Ein als sinnlos erfahrenes, aber glückliches Leben ist kaum denkbar. Auch wenn es etwas pastoral klingen mag: Sinn oder zumindest die Hoffnung auf Sinn ist dasjenige, was uns morgens aufstehen lässt, was uns schwere Zeiten durchhalten lässt, was uns schlechthin leben lässt.

Wie sehr wir auf Sinn angewiesen sind, zeigt sich vor allem dann, wenn er uns dauerhaft fehlt - oder genauer: wenn es uns an seiner Erfahrung fehlt. Die psychologische Sinnforschung zeigt, dass dann nicht nur Glück ausbleibt, sondern auch die Gesundheit Schaden nimmt, nicht zuletzt durch erhöhten Alkohol- und Drogenkonsum (vgl. Schnell 2016, S. 125-128). Auch wenn ein Mangel an Sinnerfahrung keine Krisensymptome zeigt, geht er ,einher mit subjektiver Hilflosigkeit und Kontrollverlust“" (Schnell 2016, S. 96). Vor allem aber sind Sinnkrisen oft auch Lebenskrisen, und Lebenskrisen sind vorrangig Sinnkrisen, d.h. ein als schmerzhaft und bedrohlich erlebter Mangel an Lebenssinn. Menschen in Sinnkrisen weisen eine erhöhte Neigung zur Selbsttötung auf (vgl. Schnell et al. 2018). Auch depressive Erkrankungen zeichnen sich durch die Erfahrung von Sinnlosigkeit aus und sind mit deutlich erhöhter Suizidalität verbunden (vgl. Wolfersdorf und Etzersdorfer 2011, S. 99, 147; Kleftaras und Psarra 2012). Ohne Übertreibung kann man feststellen: Sinnerfahrung und Lebensbejahung sind aufs engste verknüpft. So wie der Wunsch nach Weiterleben von einem Mindestmaß an Sinnerfahrung abhängt, so ist die Be- 
endigung des eigenen Lebens zumeist Ausdruck einer endgültigen Verneinung von Sinn. Dazu müssen sich Suizidwillige nicht die Rede von „Sinnlosigkeit“ zu eigen machen. Vielmehr kann sich dieses Urteil auch hinter anderen Begründungen verbergen, wie dem (drohenden) Verlust von Würde, Autonomie oder wichtigen sozialen Beziehungen (vgl. Van Orden et al. 2015; Bosshard 2017, S. 37 f.).

\section{Die sinnkonstituierte Verbundenheit und ein neues Verständnis von Menschenwürde}

Die Beendigung des eigenen Lebens durch Suizid, und damit auch durch assistierten Suizid, als Ausdruck einer Verneinung von Sinn - soweit mag das als Einsicht mit geringem Neuigkeitswert gelten. Doch warum sollte die Sinnverneinung eines Einzelnen für andere oder gar für alle von Relevanz sein?

Einen ersten Zugang zu diesem Gedanken können die Erfahrungen von Hinterbliebenen schaffen, die einen Angehörigen durch Suizid verloren haben. Diese Erfahrungen unterscheiden sich von der Trauer anderer Hinterbliebener. Zu dem Schmerz des Verlusts treten in vielen Fällen u. a. eine existentielle Verunsicherung, ein Gefühl des Verlassenseins, eine Infragestellung des eigenen Lebens (Jordan 2001; Tal Young et al. 2012, S. 178-180; Gamondi 2017, S. 48). Darüber hinaus haben Hinterbliebene eines Suizidenten ein deutlich höheres Risiko, psychisch zu erkranken sowie selbst Suizid zu begehen (vgl. Jordan 2001, S. 94-96; Tal Young et al. 2012, S. 181; Erlangsen et al. 2017). Die emotionalen Reaktionen und biografischen Verarbeitungsmodi der Hinterbliebenen sind natürlich individuell unterschiedlich und hängen von zahlreichen Faktoren ab. Dennoch bleibt festzuhalten, dass für viele die Selbsttötung eines nahestehenden Menschen nicht nur die Beziehung zu ihm vernichtet, sondern darüber hinaus das Sinngefüge des eigenen Lebens erschüttert. Mit anderen Worten: Die radikale Sinnverneinung des einen setzt sich teilweise im Leben der anderen fort. Das, was die eine Person ihr Leben beenden ließ, erschwert auch anderen Personen die Fortführung ihres Lebens massiv.

Dagegen drängen sich zwei Einwände auf. Erstens: Was für „,normale“ Suizide gilt, muss nicht für assistierte Suizide gelten. Die große Mehrheit von Suiziden erfolgt im Rahmen von psychischen Erkrankungen, oftmals abrupt und meist ohne Einbindung der Angehörigen. Assistierte Suizide hingegen sind ohne eine wohlüberlegte, autonome Entscheidung gar nicht zulässig und erfolgen somit in der Regel nach reiflicher Überlegung. Nicht zuletzt dadurch ist eine Einbindung von Angehörigen in den Entscheidungsprozess eher möglich und kommt auch faktisch häufiger vor. Dadurch fallen die destruktiven Folgen für die Angehörigen weitgehend weg (vgl. Gamondi 2017, S. 43 f.). - Der Einwand erscheint plausibel, tatsächlich jedoch sind die Auswirkungen assistierter Suizide auf die Angehörigen wenig erforscht. Sicherlich unterscheiden sich die Auswirkungen von Fall zu Fall und hängen von zahlreichen Faktoren ab, wie u.a. der Art der Beteiligung und der Beschaffenheit der Beziehung zu dem Suizidenten (vgl. Gamondi 2017, S. 48 f.). Es gibt Hinweise darauf, dass die Folgen nur dann weniger gravierend sind als bei anderen Suiziden, wenn die Angehörigen mit dem Suizidverlangen einverstanden waren. In den Fällen, in denen sie die Entscheidung des Betroffenen zwar aus Achtung seiner 
Autonomie respektierten, aber nicht damit einverstanden waren, scheint die spätere emotionale Situation hingegen derjenigen bei nicht-assistierten Suiziden zu ähneln (vgl. Gamondi 2017, S. 44).

Zweitens: In jedem Fall handelt es sich nicht nur um kontingente Folgen, sondern um Auswirkungen auf einen kleinen Personenkreis. Von faktischen Auswirkungen auf alle anderen kann keine Rede sein. - Der Einwand ist richtig. Die wenigsten Menschen, die Suizid begehen, kennen wir persönlich, wenn überhaupt jemanden. Wenn es eine Relevanz für alle anderen gibt, dann kann sie offensichtlich nicht in psychischen Folgen von begrenzter Reichweite liegen. Vielmehr muss es sich um eine ideelle Relevanz handeln.

Wie ließe sich eine solche ideelle Relevanz der suizidalen Sinnverneinung verstehen? Wenn die Sinnverneinung eines Menschen für alle anderen relevant sein sollte, müsste Sinn oder die Orientierung an Sinn etwas sein, in dem wir auf eine noch zu klärende Weise miteinander verbunden sind. Das mag in manchen Ohren esoterisch klingen, und dennoch lässt sich der Gedanke nüchtern plausibilisieren. Susan Wolf ist dieser Verbundenheit auf der Spur, wenn sie darüber nachdenkt, warum Sinn für uns so wichtig ist. Ihr zufolge liegt ein Teil der Antwort in unserer sozialen Natur. Es sei unser Interesse, ,to see one's life as valuable in a way that can be recognized from a point of view other than one's own" (Wolf 2010, S. 27). So unterschiedlich die individuellen Vorstellungen eines sinnvollen Lebens auch sein mögen, sind wir durch die gemeinsame Orientierung an Sinn gewissermaßen Teil einer Sinngemeinschaft:

Our interest in being able to see our lives as worthwhile from some point of view external to ourselves, and our interest in being able to see ourselves as part of an at least notional community that can understand us and that to some degree shares our point of view, then, seem to me to be pervasive, even if not universal (Wolf 2010, S. 31).

Diese Verbundenheit in einer ideellen menschlichen Gemeinschaft der Sinn-Bewertenden ist noch nicht alles. Sie hat eine Entsprechung auf axiologischer Ebene, d. h. Menschen kommt eine zentrale Rolle als sinnstiftenden Werten zu. Andere Menschen sind ganz überwiegend dasjenige, auf das in positiver Weise bezogen zu sein Sinn stiftet. Das können einzelne Menschen sein, mit denen wir in Nahbeziehungen verbunden sind. Wie gesagt dürfte diese Sinnquelle im Leben der meisten Menschen im Vordergrund stehen. Es können aber auch große Gruppen von Menschen sein, wie bei einem humanitären oder politischen Engagement. Auch bei wissenschaftlichen und ästhetischen Aktivitäten ist der Bezug auf Menschen von Bedeutung. Denn Menschen sind es, auf die ästhetische und wissenschaftliche Leistungen direkt oder indirekt abzielen: als Nutznießer, Wahrnehmende, Genießende, Miterkennende usw. Zwar ist Sinnstiftung auch durch den Bezug auf außermenschliche Werte möglich, doch erstens spielt eine solche Sinnstiftung quantitativ eine untergeordnete Rolle; und zweitens neigt ein Leben, dessen Sinn sich ausschließlich aus solchen Quellen speist, dazu, auf eigentümliche Weise verarmt zu erscheinen.

Während die Behauptung eines universalen Menschen-Bezugs bei wissenschaftlichen oder ästhetischen Projekten noch einleuchten mag, wird sie bei Nahbeziehungen auf Skepsis stoßen. Es ist doch nur die Edith, der Gregor oder die Mama, 
mit denen positiv verbunden zu sein unserem Leben Sinn verleiht. - Der Einwand ist richtig hinsichtlich dessen, worauf wir vordergründig achten. Selbstverständlich haben wir in aller Regel nicht „den“ Menschen oder die Menschheit im Blick, wenn wir etwa mit Gregor plaudern. Dennoch spielt das Menschsein für die Beziehung eine fundamentale Rolle. Man stelle sich vor, was geschähe, wenn sich Gregor eines Tages zu einem „ungeheuren Ungeziefer“ verwandeln würde (Kafka 1976, S. 56). Die vorherige Beziehung wäre zerstört. Es wäre uns unmöglich, mit dem Ungeziefer auf dieselbe Weise verbunden zu sein. Deshalb: Wenn auch normalerweise unbewusst, ist die sinnstiftende Beziehung zu einem bestimmten Menschen immer auch eine Beziehung zu einem Menschen als Menschen. Somit ist etwas von grundlegender Bedeutung, das auch allen anderen Menschen wesentlich eigen ist. Für jede partikulare sinnstiftende Beziehung zu Menschen ist das universale Moment des Menschseins notwendige Bedingung.

Menschen, und zwar Menschen überhaupt, spielen also für unsere Orientierung an Sinn eine zweifach fundamentale Rolle: als diejenigen, deren zumindest potenzielle Perspektive für das eigene Sinnstreben ausschlaggebend ist, und als diejenigen, auf die positiv bezogen zu sein unserem Leben Sinn verleiht; kurz: als Sinnbewertungsinstanz und als Sinnquelle.

Diese zentrale Bedeutung von Menschen für ein sinnvolles Leben wird auch in einem Gedankenexperiment fassbar, das Samuel Scheffler angestellt hat. Darin erfahren wir, dass kurz nach unserem eigenen natürlichen Tod die Menschheit aussterben wird. Scheffler macht plausibel, dass wir unter diesen Umständen den Sinn unserer meisten Tätigkeiten, ja unseres Lebens verloren sähen. Das ist bemerkenswert, weil es nicht das Ende des eigenen Lebens ist, auch nicht das Ende des Lebens der uns nahestehenden Menschen, das unserem Leben den Sinn raubt, sondern ,die Existenz von Menschen, die wir weder lieben noch kennen“ (Scheffler 2015, S. 56), also die Existenz von Menschen überhaupt. Er folgert:

Die Menschheit selbst, als fortdauerndes, historisches Projekt, bildet den impliziten Bezugsrahmen für den Großteil unserer Urteile darüber, was von Bedeutung ist. (Scheffler 2015, S. 78.)

Dies alles erlaubt, von einer universalen sinnkonstituierten menschlichen Verbundenheit zu sprechen. Wir sind als sinnsuchende und sinnstiftende Wesen miteinander verbunden.

Diese These dürfte auf Widerspruch stoßen. Ein erster Einwand könnte darauf verweisen, dass viele Menschen eine solche menschheitliche Verbundenheit nicht empfinden oder vielleicht sogar ausdrücklich verneinen. - Das mag so sein, trifft aber nicht die These. Die These behauptet ja keine emotionale, sondern eine ideelle Verbundenheit, die man empfinden kann, aber nicht muss. „Ideell“ heißt andererseits nicht, dass diese Verbundenheit bloß theoretischer oder imaginärer Art ist. Stattdessen handelt es sich um eine axiologische Verbundenheit, die unserer Sinnorientierung eingeschrieben ist. Wir können sie übersehen, dennoch ist sie da, kraft unserer Ausrichtung auf Sinn.

Ein zweiter Einwand hebt hervor, wie unterschiedlich unsere Sinnorientierung beschaffen ist. Die Vorstellungen eines sinnvollen Lebens unterscheiden sich von Person zu Person erheblich und schließen sich teilweise auch gegenseitig aus. Aus 
einer solchen Kakophonie partikulärer Konzeptionen lässt sich nichts Gemeinsames destillieren. - Dass sich unsere Sinnkonzeptionen massiv unterscheiden, steht außer Frage. Doch erstens kann wie gesagt nicht jede faktische Vorstellung von dem, was ein sinnvolles Leben ausmacht, als richtig gelten. Wer etwa glaubt, durch sein rassistisches Engagement seinem Leben Sinn zu verleihen, irrt sich. Und das dennoch bestehende weite Spektrum berechtigter Sinnkonzeptionen mindert nicht die ihnen gemeinsame Orientierung am Humanum. Etwas blumig formuliert: Das Universale liegt im Schatten des Partikulären. Allzu fremd dürfte diese Idee nicht sein, wie erneut ein Seitenblick auf die Moral zeigt: Auch wenn wir uns in unseren moralischen Überzeugungen unterscheiden, ist heute jede ernsthafte Moralkonzeption darauf angelegt, andere Personen als moralisch zu berücksichtigende Subjekte anzuerkennen. So wie man von der moralischen Gemeinschaft spricht - der Gemeinschaft moralischer Subjekte, die zugleich Objekte moralischer Verpflichtungen bilden -, lässt sich von der Sinngemeinschaft sprechen.

Das Verständnis der sinnkonstituierten Verbundenheit ist strukturell verwandt mit verschiedenen Ansätzen, die den Beziehungscharakter menschlichen Lebens in der ethischen Theoriebildung zur Geltung zu bringen versuchen. Dazu gehört etwa die Care-Ethik (vgl. Nodding 2013; Kittay 2020), die insbesondere auch in der Medizin- und Pflegeethik auf Widerhall stößt (vgl. Conradi und Vosman 2016). Auch jüngere Ansätze zur Begründung und Bestimmung der Menschenwürde machen das relationale Element stark (vgl. Metz 2012; Ikuenobe 2016; Kirchhoffer 2017; Miller 2017). So unterschiedlich diese Ansätze sind, treffen sie sich in dem Anliegen, die Menschenwürde in Beziehungen und Gemeinschaften oder in der Fähigkeit dazu zu verankern. Damit wollen sie eine individualistische Engführung auf die einzelne autonome Person verhindern.

Für eine ausführliche Auseinandersetzung mit diesen Ansätzen ist hier nicht der Platz. Nur so viel: Einerseits scheint es mir richtig zu sein, die Menschenwürde, die eklatant das Verhältnis zwischen Menschen betrifft, aus diesem „Zwischen“ heraus zu verstehen und zu begründen. Andererseits jedoch leiden die existierenden Ansätze teilweise unter gravierenden Begründungslücken sowie einer Tendenz zum Konservatismus, insofern sie die Gemeinschaft vor das Individuum stellen und nonkonformistischen Lebensweisen notgedrungen skeptisch begegnen müssen.

Die Sinnperspektive weist mit ihrer Betonung der Relationalität nicht nur eine strukturelle Parallele zu diesen Ansätzen auf, sondern kann selbst zu einem Verständnis der Menschenwürde ausgebaut werden, das die genannten Probleme jedoch vermeidet. Möglich ist hier nur eine grobe Skizze: Der Kern dieses Menschenwürdeverständnisses ist ebenjener Wert von Menschen, den wir, siehe oben, in unserer Orientierung am Ziel eines sinnvollen Lebens immer schon implizit voraussetzen. Die sinnkonstituierte Verbundenheit ist ja nicht eine wertfreie Relation, sondern bildet eine evaluativ gehaltvolle Gemeinschaft. In ihr ist jede menschliche Person erstens Träger einer grundsätzlichen gemeinsamen Sinnperspektive, auf die sich ein jeder Sinnsuchender implizit bezieht; zweitens eine mögliche Sinnquelle anderer Menschen; sowie drittens selbst mögliche Sinnstifterin. Die Menschenwürde $\mathrm{zu}$ achten bedeutet entsprechend, den Menschen als an Sinn orientiertes Wesen zu achten. Das heißt: ihn als gleiches Mitglied der Gemeinschaft sinnorientierter Wesen zu behandeln; ihn so behandeln, dass er als sinnstiftungsfähiges Wesen, also 
als Person zur Geltung kommt; und ihm die Möglichkeit zu geben, seine eigene Konzeption eines sinnvollen Lebens zu verwirklichen (vgl. Kipke 2020, 2021; vgl. auch Joerden 2015).

\section{Die axiologische Reichweite der suizidalen Sinnverneinung}

Wenn die Überlegungen zur sinnkonstituierten Verbundenheit und der so begründeten Menschenwürde richtig sind, wirft das ein neues Licht auf den Suizid und seine Unterstützung. Denn wenn ein Suizid Ausdruck einer endgültigen Verneinung von Sinn ist und wir alle durch diese Dimension des Sinns verbunden sind, geht dieser Suizid uns alle an. Ein Suizid verneint - zumeist - in grundlegender Weise dasjenige, was unser Leben lebenswert macht, und dies auf radikale und irreversible Weise. Natürlich sind es jeweils ganz verschiedene Aktivitäten, Projekte, Mitmenschen, die ein Leben lebenswert machen oder zumindest machen könnten. Dennoch verneint ein Suizid nicht nur die konkreten potenziellen Sinnquellen der jeweiligen Person, sondern immer auch die Möglichkeit von Lebenssinn selbst. Bildhaft ausgedrückt: Ein Suizid zerschneidet das gemeinsame Netz, das uns trägt, auch wenn dieses Netz aus sehr unterschiedlichen Materialien geflochten ist. Somit gilt das, was viele Angehörige, die einen Menschen durch Suizid verloren haben, emotional erfahren, auf einer axiologischen Ebene für alle Menschen. Ein Suizid macht nur einige betroffen, doch seine sinnverneinende Geste betrifft uns alle.

Wenn dieser Gedanke befremdlich erscheint, liegt es an der Gewohnheit, uns nur als autonome Individuen zu betrachten, die ihre eigenen Vorstellungen sinnvollen Lebens entwickeln und zu verwirklichen versuchen. Diese Autonomie ist ja in der Tat ein enorm wichtiger und normativ relevanter Aspekt unseres Lebens. Aber sie ist bei weitem nicht alles, was sich über personales Handeln und insbesondere über das Streben nach einem sinnvollen Leben sagen lässt. Ein vernachlässigter Aspekt besteht darin, dass wir in unserem individuellen Sinnstreben auf eine untergründige Weise aufeinander bezogen und voneinander abhängig sind. Und wer sich aus Gründen der Sinnverneinung selbst tötet, kappt diese Verbindung und verneint damit nolens volens nicht nur sein eigenes Leben.

Diese Verletzung der sinnkonstituierten Verbundenheit wird vielleicht einsichtiger, wenn wir sie in der vertrauteren Begrifflichkeit der Menschenwürde reformulieren. Damit wird zugleich die normative Ambivalenz des assistierten Suizids deutlicher: Eine im Sozialen gründende, auf unserer Orientierung an Sinn bauende Konzeption der Menschenwürde kann nicht darüber hinwegsehen, wenn Menschen sich selbst vernichten. Denn damit wird die Grundlage der Menschenwürde in Frage gestellt. Zugleich gehört der Schutz autonomer Lebensführung, die im Extremfall einen Suizid einschließen kann, zum Kern der Menschenwürdegarantie. Es ist nur scheinbar paradox: Zu einer am Prinzip der Menschenwürde orientierten Rechtsordnung gehört es selbstverständlich, Suizide nicht zu pönalisieren. Ebenso richtig ist aber, dass eine Gesellschaft, die die Menschenwürde ernst nimmt und nicht auf Autonomieachtung reduziert, mit der Unterstützung von Suiziden hadern muss nicht trotz der Menschenwürde, sondern um der Menschenwürde willen. 
Soweit zur sinnkonstituierten Verbundenheit und der dadurch sich ergebenden gesellschaftlichen Relevanz des Suizids. Abkürzend spreche ich im Folgenden von der „Sinnperspektive auf den (assistierten) Suizid“ oder kurz von der „Sinnperspektive“. Was aber ist mit der Sinnperspektive gewonnen?

\section{Die Vorzüge der Sinnperspektive auf den assistierten Suizid}

Gewiss, die Sinnperspektive auf den assistierten Suizid ist ungewöhnlich. Die Qualität eines ethischen Ansatzes bemisst sich jedoch nicht am Grad seiner Gewöhnlichkeit, sondern vor allem daran, ob er moralische Urteile verständlich machen und begründen kann. Genau das leistet die Sinnperspektive.

Erstens macht die Sinnperspektive die Bedeutung des Suizids und damit des assistierten Suizids überhaupt erst verständlich. Was ist damit gemeint? Nach Johannes Fischer ist die Bedeutung von Handlungen und Ereignissen ein wesentliches Moment moralischen Urteilens, das jedoch in der akademischen Ethik vernachlässigt wird (Fischer 2009). Gemeint ist dasjenige, was uns Handlungen und Ereignisse bedeuten, welches Gewicht sie für uns haben, welchen Platz sie im Gefüge unserer evaluativ gefüllten Weltwahrnehmung einnehmen, jenseits eines verfehlten ,Dualismus von Tatsachen und Wertungen“ (Fischer 2009, S. 246). Erst vor dem Hintergrund dieser Bedeutung werden die emotionalen Reaktionen verständlich, wie zum Beispiel Betroffenheit. Und diese Bedeutung ist auch für die moralische Beurteilung ausschlaggebend.

Fischer weist ganz richtig darauf hin, dass die Bedeutung des Suizids in der ethischen Debatte theoretisch unterbelichtet ist. Die Sinnperspektive erschließt diese Bedeutung des Suizids. Sie vermag die Betroffenheit, die Verstörung verständlich zu machen, die wir empfinden können, wenn wir von einem Suizid auch einer entfernt bekannten oder unbekannten Person hören. Insbesondere kann sie verständlich machen, warum und inwiefern uns ein Suizid etwas angeht, und zwar nicht nur dann, wenn wir mit der Person vertraut waren.

Genauer muss man sagen, dass die Sinnperspektive einen Teil der Bedeutung des Suizids verständlich macht, vor allem des assistierten Suizids. Einen anderen Teil dieser Bedeutung bildet nämlich die Autonomie der suizidalen Entscheidung. Das heißt, das, was ein assistierter Suizid bedeutet, liegt auch maßgeblich darin, dass sich ein zu selbstbestimmten Entscheidungen fähiger Mensch entschlossen hat, seinem Leben ein Ende zu setzen. Erst aus dieser Spannung zwischen der uns betreffenden Sinnverneinung und der zu achtenden individuellen Autonomie wird die soziale Problematik und moralische Konfliktträchtigkeit des assistierten Suizids verständlich. Diese Spannung geht im ethischen Diskurs zuweilen unter, wenn die Suizidthematik auf die Autonomiefrage reduziert wird, ,so als ginge ein Suizid gewissermaßen ,in Ordnung', wenn die psychiatrische Abklärung ergeben hat, dass er freiverantwortlich und selbstbestimmt ist" (Fischer 2009, S. 245).

Zweitens kann die Sinnperspektive die prekäre Rolle der Menschenwürde in der Diskussion um den assistierten Suizid aufklären. Bekanntlich wird die Menschenwürde für gegensätzliche Positionen ins Feld geführt: für und wider den assistierten Suizid (vgl. Borasio et al. 2020, S. 86f.; Wittwer 2020, S. 186-196). Die Sinnper- 
spektive zeigt nun, dass der Grund für diesen merkwürdigen Status der Menschenwürdeidee keineswegs in einer inhaltlichen Beliebigkeit liegt, sondern in ihrem klar fassbaren, aber mehrdimensionalen Gehalt. Die Kontrahenten der Debatte nehmen auf verschiedene Dimensionen der Menschenwürde Bezug. Die Pflicht zur Achtung selbstbestimmter Suizidentscheidungen folgt aus ihr ebenso wie eine kritische Bewertung ihrer Unterstützung. Suizidassistenz verletzt nicht die Menschenwürde, aber sie untergräbt ihre axiologische Grundlage. Das macht diese Handlungsweise ethisch so irritierend. Jede einseitige Reduktion auf nur einen Aspekt wird der Menschenwürde nicht gerecht. Die sinntheoretische Aufdeckung dieser Spannung macht die Bewertung des assistierten Suizids nicht einfacher, aber sie wird seiner normativ-evaluativen Komplexität gerechter.

Drittens gelingt es der Sinnperspektive, die unterschiedlichen Bewertungen verschiedener Suizidgründe verständlich zu machen. Beim klassischen Fall des assistierten Suizids handelt es sich um Menschen mit tödlichen Erkrankungen. Inzwischen wird jedoch Suizidassistenz ebenso bei „Lebensmüdigkeit“" gerechtfertigt und praktiziert. Auch das Bundesverfassungsgericht bahnt dafür den Weg (BVerfG 2020, Rn. 210). Während die Suizidassistenz bei Sterbenskranken vielfach auf Verständnis stößt, ruft sie im Falle Lebensmüder eher Skepsis hervor. Warum? Versteht man Suizidassistenz nur als Hilfe bei der Umsetzung eines autonomen Wunsches in existentieller Not, ist diese unterschiedliche Bewertung nicht nachvollziehbar. In der Sinnperspektive ändert sich das. Ein Suizid aus Gründen der Lebensmüdigkeit ist der geradezu paradigmatische Fall der Sinnverneinung: Eine Person beendet ihr Leben, eben weil es in ihren Augen sinnlos ist oder geworden ist. Beim Suizid aus krankheitsbedingten Gründen hingegen kann neben der Sinnverneinung ein gewisses Maß an Sinnbejahung vorhanden sein, zum Beispiel in der Bejahung wichtiger Nahbeziehungen, die sogar in die Realisierung des Suizidvorhabens involviert sein können. Das lässt solch einen assistierten Suizid in einem partiell anderen Lichte erscheinen. Nur partiell, weil der durch die Erkrankung oder das erwartete Leid erzeugte Verlust an Sinnerfahrung die bestehende Sinnbejahung letztlich überlagert und sich im Suizid endgültig ausdrückt.

Nochmal anders verhält es sich bei einem Suizid von jemandem, der nicht lebensmüde, sondern ,lebenssatt“ ist, wie man so sagt. Wer sein Leben als sinnvoll bejaht und gerade wegen des Sinnreichtums dieses Lebens in fortgeschrittenem Alter als gerundet und beendigungswürdig ansieht, dürfte auf breites Verständnis stoßen. Die Sinnperspektive weiß warum: Der Suizid(wunsch) verliert hier weitgehend seinen sinnverneinenden Stachel.

Schließlich kann die Sinnperspektive auch die besonderen Bewertungen verständlich machen, die sich mit den seltenen Fällen von Suiziden als Selbstopfer verbinden. $\mathrm{Zu}$ denken ist etwa an die Selbstverbrennung als Protest gegen ein Unrechtsregime. Während Suizide sonst allenfalls auf Verständnis stoßen, rufen solche altruistischen Suizide Reaktionen wie Bewunderung hervor. Nicht überraschend, denn sie dienen einem wertvollen Zweck jenseits des eigenen Wohls, d. h. sie stiften selbst Sinn (vgl. Metz 2016, S. 30 f.).

Während wir also unter alleiniger Maßgabe des Respekt vor der Autonomie gezwungen sind, sämtliche Suizide und Suizidwünsche ethisch über einen Kamm zu scheren, sofern nur die jeweilige Entscheidung hinreichend autonom ist, ermöglicht 
die Suizidperspektive vielfältige Differenzierungen. Die unterschiedlichen Bewertungen und Ambivalenzen, die sonst als ideologische Relikte oder widersprüchliche, nicht öffentlich rechtfertigbare Intuitionen erscheinen, kann die Sinnperspektive begreiflich machen - ohne die normative Relevanz der Autonomie dabei vernachlässigen zu müssen.

Viertens: Erst die sich in der Sinnperspektive erschließende Bedeutung des Suizids kann das Primat der Suizidprävention begründen. Für die Mehrzahl der Suizidvorhaben fällt eine solche Begründung zwar nicht schwer, weil hier die Fähigkeit zum rationalen, selbstbestimmten Handeln als stark eingeschränkt gilt. Aber Verteidiger des Rechts auf Suizidassistenz betonen, dass auch freiverantwortliche Suizide nach Möglichkeit zu vermeiden sind (vgl. Deutscher Ethikrat 2014; Neitzke et al. 2013, S. 167, 169; Merkel 2015, S. 6). Doch warum? Was geht es uns an, wenn sich Menschen selbstbestimmt für die Selbsttötung entscheiden? Warum sollten wir trotz letztlicher Achtung ihrer Autonomie zunächst versuchen, sie davon abzubringen, und ihnen eine ,lebensorientierte Beratung“ zukommen lassen (Deutscher Ethikrat 2014; vgl. Borasio et al. 2020, S. 96)? Die nahezu konsensuale Auffassung, dass die Unterstützung beim Suizid nur ultima ratio sein darf und dass der Suizidvermeidung Vorrang zukommt, steht in auffälligem Kontrast zu ihrer weithin fehlenden ethischen Begründung. Erstaunlich jedoch ist das nicht. Denn eine solche Begründung ist innerhalb eines ethischen Rahmens, der bei der Bedeutung des Suizids ausschließlich die Seite der Autonomie gelten lässt und sich ansonsten auf ethische Neutralität beruft, nicht möglich. Eine solche Begründung müsste einräumen, dass der Suizid in der Regel ein Übel ist, das möglichst verhindert werden sollte (vgl. Kipke 2019, S. 317). Doch diese Wertung steht im einseitigen Autonomieparadigma unweigerlich unter Paternalismusverdacht und wird daher zumeist sorgsam vermieden.

Lässt sich das Primat der Suizidprävention nicht viel leichter durch das Prinzip des Lebensschutzes rechtfertigen? Im bundesdeutschen Verfassungsrecht jedenfalls ist es üblich, das Recht auf Leben nicht nur im Sinne eines subjektiven Abwehrrechts, sondern auch als objektive Garantie für das Rechtsgut Leben zu begreifen. - Für die Verhinderung nicht-freiverantwortlicher Suizide ist das sicherlich ausreichend. Doch sobald die Freiverantwortlichkeit eines Suizidwunsches gewiss ist, droht der Lebensschutz ein ,gegen die Autonomie gerichteter Lebensschutz“ zu werden, wie ihn das Bundesverfassungsgericht in seiner Entscheidung vom 26.02.2020 ausdrücklich verworfen hat (BVerfG 2020, Rn. 277). Vor demselben Problem steht der Versuch, Suizidprävention mit der Irreversibilität der Selbsttötung zu begründen. Denn die Irreversibilität ist innerhalb des einseitigen Autonomieparadigmas nur so lange ein guter Grund, jemanden von seinem Suizidwunsch abzubringen, wie die Autonomie dieses Wunsches nicht feststeht. Sobald das jedoch der Fall ist, müssen präventive Bemühungen mit Verweis auf die Irreversibilität als paternalistisch gelten.

Die Sinnperspektive hingegen ist in der Lage, dem für selbstverständlich gehaltenen Primat der Suizidprävention eine Rechtfertigungsgrundlage zu verschaffen. In dieser Perspektive nämlich ist die Selbsttötung nichts, was allein die suizidale Person etwas angeht. Vielmehr betrifft die suizidale Sinn- und Lebensverneinung der einen Person auch jede andere Person. Die beabsichtigte Selbstvernichtung wäre eine Infragestellung der evaluativen und normativen Grundlagen unseres Zusammenlebens, ob wir das so empfinden oder nicht. 
Dass es hier nicht um unverbindliche Idiosynkrasien zart besaiteter Gemüter geht, sondern um vitale und berechtigte Interessen der Rechtsgemeinschaft, wird spätestens dann deutlich, wenn wir die sinnkonstituierte Verbundenheit menschenwürdetheoretisch reformulieren. Der freiverantwortliche Suizid fällt einerseits in den Bereich höchstpersönlicher Entscheidungen, der durch den Menschenwürdegrundsatz geschützt ist, und untergräbt andererseits die axiologische Basis eben dieser Menschenwürde. Daher muss die menschenwürdebasierte Rechtsgemeinschaft daran interessiert sein, um der Menschenwürde willen Suizide nach Möglichkeit zu verhindern, so wie sie ebendiese Suizide um der Menschenwürde willen akzeptieren muss. Deshalb sind Präventionsversuche bei Personen mit freiverantwortlichen Entscheidungen gerechtfertigt, auch wenn letztendlich ihre gegebenenfalls unerschütterliche Suizidabsicht zu respektieren ist.

Fünftens kann die Sinnperspektive begründen, warum Ärzte das Recht zur Verweigerung von Suizidassistenz haben sollten. Auch dies ist eine verbreitete Position, die ebenfalls bislang auf tönernen Füßen steht. Das Bundesverfassungsgericht erklärt in seinem Urteil vom 26. Februar 2020 apodiktisch: ,Die mangelnde individuelle ärztliche Bereitschaft zur Suizidhilfe hat der Einzelne als durch die Gewissensfreiheit seines Gegenübers geschützte Entscheidung grundsätzlich hinzunehmen“ (BVerfG 2020, Rn. 289; vgl. Merkel 2015, S. 6). Doch warum sollten Ärzte das Recht haben, eine Handlung aus Gewissensgründen zu verweigern, um die jemand nicht nur aufgrund einer selbstbestimmten Entscheidung bittet, sondern die zudem als Hilfe in äußerster Not gilt? Die Frage wird umso dringlicher, als Ärzte privilegierten Zugang zu den tödlichen Mitteln haben, Verweigerungen von ihrer Seite also die Umsetzung eines Suizidwunschs erheblich erschweren.

Die gängige Antwort lautet: Das Recht auf assistierten Suizid ist ein Abwehrrecht und kein Anspruchsrecht. Doch diese Antwort trägt zur gesuchten Begründung nichts bei, sondern ist lediglich die komplementäre Formulierung dazu, dass Ärzte das Recht haben, die Unterstützung beim Suizid abzulehnen. Genau diese Beschränkung auf ein Abwehrrecht ist zu begründen. Eine solche Begründung ist aber nicht in Sicht, solange die Entscheidung für den Suizid nur als individuelle autonome Entscheidung in einer Notsituation verstanden wird. Denn warum sollten wir den Anspruch auf ärztliche Hilfe bei Kreuzbandriss und Fußpilz haben, während solch ein Anspruch in einer weitaus dramatischeren Notlage ausgeschlossen ist? Die Beschränkung auf ein Abwehrrecht bei der Suizidassistenz ist unter Maßgabe üblicher normativer Annahmen schlicht inkohärent.

Auch der Verweis auf das ärztliche Gewissen hilft nicht weiter (vgl. z.B. Borasio et al. 2020, S. 97; Wittwer 2020, S. 226). Denn die ärztliche Gewissensfreiheit reicht grundsätzlich nicht beliebig weit, sondern ist moralisch und rechtlich stets dem Patienten verpflichtet (vgl. Duttge 2014). Auch speziell die ärztliche Verweigerung aus Gewissensgründen ist an übergeordnete Regeln gebunden. Wir würden ja auch nicht akzeptieren, wenn ein Arzt empfohlene Impfungen oder die Behandlung von Angehörigen des anderen Geschlechts unter Berufung auf sein Gewissen verweigern würde. Konsequenterweise wird ein ärztliches Recht auf Verweigerung aus Gewissensgründen in der jüngeren medizinethischen Diskussion vielfach verneint, auch gerade mit Bezug auf die Suizidassistenz (vgl. Giubilini 2014; Cholbi 2015; Schuklenk und Smalling 2017). Cholbi schreibt exemplarisch: „In denying patients 
such access, these conscientious objectors exploit their role as monopolists in the service of a kind of moral paternalism, i.e., they compel patients to live according to the physician's conception of the good" (Cholbi 2015, S. 492). Erneut sieht sich eine heute noch übliche medizinethische Position in Fragen des assistierten Suizids dem Vorwurf des Paternalismus ausgesetzt. Das ist zwangsläufig so, wenn Suizidassistenz lediglich als Unterstützung bei der Selbstbestimmung in einer Notsituation verstanden wird.

Doch ist die ärztliche Gewissensfreiheit als Mitwirkungsverweigerungsrecht nicht an anderer Stelle, nämlich zumindest beim Schwangerschaftsabbruch rechtlich verankert und gesellschaftlich akzeptiert? Ja. (Kritisch dazu: Hillenkamp 2010.) Aber abgesehen davon, dass aktuelle Unumstrittenheit und gesetzliche Erlaubnis kein Ausweis für ethische Konsistenz sind: Erstens gilt dieses Mitwirkungsverweigerungsrecht keineswegs unter allen Umständen (vgl. § 12 Abs. 2 Schwangerschaftskonfliktgesetz). Zweitens und vor allem geht es hier um die gezielte Tötung eines werdenden menschlichen Lebens ohne dessen Einwilligung. Das eventuell vorhandene Lebensschutzinteresse seitens des Arztes richtet sich nicht auf die schwangere Frau, die den Eingriff wünscht, sondern auf ein anderes menschliches Wesen. Das kann eine Verweigerung aus Gewissensgründen rechtfertigen. Anders beim assistierten Suizid: Hier entscheidet sich eine Person autonom für den eigenen Tod und bittet dafür um Unterstützung. Ein ärztlicherseits eventuell in Anschlag gebrachtes Lebensschutzinteresse richtet sich auf dieselbe Person, die um die Suizidassistenz ersucht - und damit unmittelbar gegen deren Autonomie. Denn hier wird nicht, wie bei der Frage nach einem Schwangerschaftsabbruch, die Autonomie des einen gegen das Leben des anderen abgewogen. Vielmehr wird die Lebensschutzorientierung der sterbewilligen Person gewissermaßen oktroyiert und damit ihr autonomer Sterbewunsch desavouiert - und dies in einer Situation größter Not. Eine ärztliche Verweigerung aus Gewissensgründen angesichts eines autonomen Suizidwunsches fällt somit zwangsläufig unter das Verdikt des Paternalismus. So stellt es sich jedenfalls im Rahmen einer ausschließlich autonomie-zentrierten Ethik des assistierten Suizids dar. Es bleibt also dabei: Eine solche Ethik hat keine Kapazitäten zur Begründung eines ärztlichen Rechts, die Mitwirkung am Suizid zu verweigern.

Die Sinnperspektive kann aus dieser begründungstheoretischen Sackgasse herausführen. Denn sie macht deutlich, dass der individuelle Suizid eine gesellschaftliche Angelegenheit und von Übel ist. Die Verneinung des Lebenssinns betrifft uns alle, weil wir allesamt auf diese axiologische Ressource angewiesen sind. Die nihilistische Geste des Suizids kratzt an den Grundlagen unserer normativen, auch rechtlichen Ordnung. Die Weigerung, an einem Suizid mitzuwirken, muss deshalb nicht eine fragwürdige Gewissensentscheidung mit paternalistischer Schlagseite sein, sondern kann Ausdruck des Bewusstseins für die sinnkonstituierte Verbundenheit sein, in der unsere normative Ordnung wurzelt.

Mit alledem lässt die Sinnperspektive - sechstens - verständlicher werden, inwiefern auf den verschiedenen Seiten des Disputes humane Motivationen stehen. Suizidassistenz ist heftig umkämpft wie wohl kaum ein anderes Thema innerhalb der Medizinethik. Die Auseinandersetzung ist auch von gegenseitigen moralischen Vorwürfen geprägt: wahlweise paternalistische Kaltherzigkeit gegenüber den suizidwilligen Menschen oder Rücksichtslosigkeit gegenüber jenen, die auf Zuspruch und 
Fürsorge angewiesen sind. Die Sinnperspektive löst diese unersprießliche Frontstellung auf und bietet eine Grundlage für die ethische Verständigung.

Wir sehen: Die Sinnperspektive hat erhebliches Potenzial zur Aufklärung und Begründung normativer und evaluativer Annahmen, die in der Debatte um den assistierten Suizid eine zentrale Rolle spielen. Sie vermeidet dabei systematisch übliche normative Einseitigkeiten und wird so der Ambivalenz und dem Grenzfallcharakter des assistierten Suizids gerecht. ${ }^{2}$

Funding Open Access funding enabled and organized by Projekt DEAL.

Open Access Dieser Artikel wird unter der Creative Commons Namensnennung 4.0 International Lizenz veröffentlicht, welche die Nutzung, Vervielfältigung, Bearbeitung, Verbreitung und Wiedergabe in jeglichem Medium und Format erlaubt, sofern Sie den/die ursprünglichen Autor(en) und die Quelle ordnungsgemäß nennen, einen Link zur Creative Commons Lizenz beifügen und angeben, ob Änderungen vorgenommen wurden.

Die in diesem Artikel enthaltenen Bilder und sonstiges Drittmaterial unterliegen ebenfalls der genannten Creative Commons Lizenz, sofern sich aus der Abbildungslegende nichts anderes ergibt. Sofern das betreffende Material nicht unter der genannten Creative Commons Lizenz steht und die betreffende Handlung nicht nach gesetzlichen Vorschriften erlaubt ist, ist für die oben aufgeführten Weiterverwendungen des Materials die Einwilligung des jeweiligen Rechteinhabers einzuholen.

Weitere Details zur Lizenz entnehmen Sie bitte der Lizenzinformation auf http://creativecommons.org/ licenses/by/4.0/deed.de.

\section{Einhaltung ethischer Richtlinien}

Interessenkonflikt R. Kipke gibt an, dass kein Interessenkonflikt besteht.

Ethische Standards Für diesen Beitrag wurden von dem Autor keine Studien an Menschen oder Tieren durchgeführt. Für die aufgeführten Studien gelten die jeweils dort angegebenen ethischen Richtlinien.

\section{Literatur}

Ach JS, Schöne-Seifert B (2013) „Relationale Autonomie“. Eine kritische Analyse. In: Wiesemann C, Simon A (Hrsg) Patientenautonomie. Theoretische Grundlagen, Praktische Anwendungen. mentis, Münster, S 42-60

Beauchamp TL, Childress JF (2019) Principles of biomedical ethics, 8. Aufl. Oxford University Press, New York, Oxford

Borasio GD, Jox RJ, Taupitz J, Wiesing U (2020) Selbstbestimmung im Sterben - Fürsorge zum Leben. Ein verfassungskonformer Gesetzesvorschlag zur Regelung des assistierten Suizids, 2. Aufl. Kohlhammer, Stuttgart

Bosshard G (2017) Assistierter Suizid in der Schweiz: Ursprung, Entwicklungen, empirische Befunde. In: Borasio GD, Jox RJ, Taupitz J, Wiesing U (Hrsg) Assistierter Suizid. Der Stand der Wissenschaft mit einem Kommentar zum neuen Sterbehilfe-Gesetz. Springer, Berlin, Heidelberg, S 29-40

Bundesverfassungsgericht (BVerfG) (2020) Urteil des Zweiten Senats vom 26. Februar 2020, 2 BvR 2347/15, Rn. 1-343. www.bverfg.de/e/rs20200226_2bvr234715.html. Zugegriffen: 3. August 2021

Cholbi M (2015) The right to die and the medical cartel. Ethics Med Public Health 1:486-493

Conradi E, Vosman F (Hrsg) (2016) Praxis der Achtsamkeit. Schlüsselbegriffe der Care-Ethik. Campus, Frankfurt a.M.

\footnotetext{
${ }^{2}$ Ich danke Markus Rüther, Peter Schaber und den zwei anonymen Gutachtern/innen für wertvolle Hinweise.
} 
Deutscher Ethikrat (2014) Zur Regelung der Suizidbeihilfe in einer offenen Gesellschaft. Deutscher Ethikrat empfiehlt gesetzliche Stärkung der Suizidprävention. Ad-hoc-Empfehlung. www.ethikrat. org/fileadmin/Publikationen/Ad-hoc-Empfehlungen/deutsch/empfehlung-suizidbeihilfe.pdf. Zugegriffen: 29. Juli 2021

Duttge G (2014) Das Gewissen im Kontext des modernen Arztrechts. In: Bormann FJ, Wetzstein V (Hrsg) Gewissen. Dimensionen eines Grundbegriffs medizinischer Ethik. De Gruyter, Berlin, Boston, S 543-560

Erlangsen A, Runeson B, Bolton JM, Wilcox HC, Forman JL, Krogh J et al (2017) Association between spousal suicide and mental, physical, and social health outcomes: a longitudinal and nationwide register-based study. JAMA Psychiatry 74:456-464

Fischer J (2009) Warum überhaupt ist Suizid ein ethisches Problem? Über Suizid und Suizidbeihilfe. Z Med Ethik 55(3):243-256

Gamondi C (2017) Relatives' experiences in assisted suicide decision-making: Overview of the literature with specific focus on the Swiss experience. In: Borasio GD, Jox RJ, Taupitz J, Wiesing U (Hrsg) Assistierter Suizid. Der Stand der Wissenschaft mit einem Kommentar zum neuen Sterbehilfe-Gesetz. Springer, Berlin, Heidelberg, S 41-50

Giubilini A (2014) The paradox of conscientious objection and the anemic concept of „,conscience“: Downplaying the role of moral integrity in health care. Kennedy Inst Ethics J 24(2):159-185

Gutmann T (2002) Der eigene Tod - Die Selbstbestimmung des Patienten und der Schutz des Lebens in ethischer und rechtlicher Dimension. Ethik Med 14:170-185

Hillenkamp T (2010) Zum Mitwirkungsverweigerungsrecht beim Schwangerschaftsabbruch. In: Dölling D, Götting B, Meier BD, Verrel T (Hrsg) Verbrechen - Strafe - Resozialisierung. Festschrift für Heinz Schöch zum 70. Geburtstag am 20. August 2010. De Gruyter, Berlin, S 511-530

Ikuenobe PA (2016) The communal basis for moral dignity. An African perspective. Philos Pap 45(3):437-469

Joerden JC (2015) Menschenwürdeschutz und Sinnstiftung. In: Brockmöller A, Kirste S, Neumann U (Hrsg) Wert und Wahrheit in der Rechtswissenschaft. Im Gedenken an Gerhard Sprenger. Franz Steiner/Nomos, Stuttgart, S 75-84

Jordan JR (2001) Is suicide bereavement different? A reassessment of the literature. Suicide Life Threat Behav 31(1):91-102

Kafka F (1976) Die Verwandlung. In: Kafka F (Hrsg) Sämtliche Erzählungen. Fischer, Frankfurt a.M., S 56-99

Kipke R (2019) Scheinneutralität. Über einen Vorschlag zur Regelung des assistierten Suizids und die Frage nach der Legitimität seines gesetzlichen Verbots. In: Mitscherlich-Schönherr O (Hrsg) Gelingendes Sterben. Zeitgenössische Theorien im interdisziplinären Dialog. De Gruyter, Berlin/Boston, S 299-325

Kipke R (2020) Die Sinntheorie der Menschenwürde. Auf dem Weg zu einem neuen und integrativen Ansatz. Z Prakt Philos 7(2):91-118

Kipke R (2021) Inwiefern verletzt Folter die Menschenwürde? Zwei Antworten im Vergleich. In: Kipke R, Röttger N, Wagner J, von Wedelstaedt AK (Hrsg) ZusammenDenken. Festschrift für Ralf Stoecker. Springer VS, Wiesbaden, S 205-226

Kirchhoffer DG (2017) Human dignity and human enhancement. A multidimensional approach. Bioethics 31(5):375-383

Kittay EF (2020) Love's labor. Essays on women, equality, and dependency, 2. Aufl. Routledge, New York

Kleftaras G, Psarra E (2012) Meaning in life, psychological well-being and depressive symptomatology: a comparative study. Psychology 3(4):337-345

Landau I (2017) Finding meaning in an imperfect world. Oxford University Press, Oxford

Merkel R (2015) Stellungnahme für die öffentliche Anhörung am 23.09.2015 im Ausschuss des Bundestages für Recht und Verbraucherschutz. www.bundestag.de/blob/388404/ ad20696aca7464874fd19e2dd93933c1/merkeldata.pdf. Zugegriffen: 30. Jan. 2021

Metz T (2012) African conceptions of human dignity. Vitality and community as the ground of human rights. Hum Rights Rev 13:19-37

Metz T (2015) Meaning in life. An analytic study. Oxford University Press, Oxford

Metz T (2016) The proper aim of therapy: Subjective well-being, objective goodness, or a meaningful life? In: Russo-Netzer P, Schulenberg SE, Batthyany A (Hrsg) Clinical perspectives on meaning. Positive and existential psychotherapy. Springer, Cham, S 17-35

Miller SC (2017) Reconsidering dignity relationally. Ethics Soc Welf 11(2):108-121 
Neitzke G, Coors M, Diemer W, Holtappels P, Spittler JF, Wördehoff D (2013) Empfehlungen zum Umgang mit dem Wunsch nach Suizidhilfe. Arbeitsgruppe „Ethik am Lebensende“ in der Akademie für Ethik in der Medizin e. V. (AEM). Ethik Med 25:349-365

Noddings N (2013) Caring. A relational approach to ethics and moral education, 2. Aufl. University of California Press, Berkeley

Van Orden KA, Wiktorsson S, Duberstein P, Berg AI, Fässberg MM, Waern M (2015) Reasons for attempted suicide in later life. Am J Geriatr Psychiatry 23(5):536-544

Rüther M (2021) Meaning in Life oder: Die Debatte um das sinnvolle Leben - Überblick über ein neues Forschungsthema in der analytischen Ethik. Teil 2: normativ-inhaltliche Fragen. Z Phil Forsch 75(2):316-354

Scheffler S (2015) Der Tod und das Leben danach. Suhrkamp, Berlin

Schnell T (2016) Psychologie des Lebenssinns. Springer, Berlin/Heidelberg

Schnell T, Gerstner R, Krampe H (2018) Crisis of meaning predicts suicidality in youth independently of depression. Crisis 39:294-303

Schuklenk U, Smalling R (2017) Why medical professionals have no moral claim to conscientious objection accommodation in liberal democracies. J Med Ethics 43:234-240

Tal Young I, Iglewicz A, Glorioso D, Lanouette N, Seay K, Ilapakurti M, Zisook S (2012) Suicide bereavement and complicated grief. Dialogues Clin Neurosci 14(2):177-186

Wittwer H (2020) Das Leben beenden. Über die Ethik der Selbsttötung. mentis, Paderborn

Wolf S (2010) Meaning in life and why it matters. Princeton University Press, Princeton

Wolfersdorf M, Etzersdorfer E (2011) Suizid und Suizidprävention. Kohlhammer, Stuttgart 